\title{
L'ONOMASTIQUE ESPAGNOLE EN TUNISIE
}

Por

NABILA ZBISS

"Enfin, chacun de nous s'inscrit par son nom dans un système de filiation qui marque son appartenance à une lignée juridiquement et culturellement marque»... la trasmission du patronyme (J. Lacan, G. Rosolato, P. Legendre) est l'élément essentiel qui fonde notre identité et nous inscrit dans le symbolique, mais le nom du père est aussi en un autre sens, ce qui assigne l'origine, il est ainsi porteur d'histoire, évocateur d'une éthnie ou d'une région et parfois signifiant dans la langue maternelle...».

Emmanuelle DIET, «Je et l'autre», Enfances et Cultures. A.N.P.A.S.E. Privat, p. 66

\section{Les Espagnols musulmans amenèrent en Tunisie une onomastique riche et éthniquement très variée}

Aux premiers siècles de l'islam, la Tunisie et l'Espagne constituaient une même terre musulmane dans laquelle on pouvait circuler à loisir et même s'y ètablir à demeure.

Kairouan par exemple était un lieu de culture ifriquien par excellence et elle était trés appréciée par les Andalous qui, à l'allée et au retour du pélerinage, ou d'une expédition commerciale, ou à la recherche d'un bon maître spirituel, s'y arrêtaient plus ou moins longuement ou y établissaient domicile jusqu'à la mort.

C'est le cas par exemple de Yahya Ibn Umar, aujourd'hui saint-patron de la ville de Sousse, qui avait réuni autour de lui une pléiade de chei'khs d'Al-Andalus, venus étudier à Kairouan. C'est aussi le cas de Abou Midien Chouaïb mort à Tlemcen, qui s'était arrêté longtemps à Tunis pour enseigner le soufisme à un groupe de chei'khs tunisiens. 
Au 13 eme siècle, quelques temps avant et après la chute de Séville, de Cordoue et de Valence entraient à Tunis un aréopage considérable d'Espagnols brillants: médecins, hommes de lettres, grammairiens, historiens, traditionnistes et profonds connaisseurs des sciences du Coran.

Ces personnages venaient de diverses villes d'Espagne et s'attachaient strictement à leur éthnique. Ils étaient avant tout Sévillans, Almériens, Valenciens, Corduans, etc.

A partir des derniers exodes consécutifs aux édits de 1609 et des années suivantes, nous allons avoir en Tunisie des musulmans qui, pendant un siècle, avaient subi les pires vexations, tendant à les christianiser de force avec suppression de leurs noms arabes et l'adoption de nouveaux noms chrétiens, ainsi que l'interdiction stricte de la langue arabe, de fréquenter les hammams et de se vêtir à la mode de leurs ancêtres.

Nous allons avoir des gens qui s'appellent: Marcou, Bantor, Finnira, Zapata, Madhor, Manachou, Rchicou, Boutricou, etc.

Quant aux noms éthniques, ils représentent la presque totalité de l'Espagne et du Portugal. A partir des derniers exodes, les noms éthniques changent de forme: on n'est plus Valencien, «Balancianou», Cordouan, «Cortobi», Sévillan, «lchbili»... Le «de» disparait et l'on prend directement le nom de la ville ou du village d'où l'on vient (les Teruel, les Ouichca, les Carabaca, les Chouria, etc..., au lieu de: de Teruel, de Huesca, de Caravaca, de Soria).

Les Andalous descendus à Tunis, avaient rempli tous les quartiers capables de les accueillir, et avaient formé un groupe de responsables qui étaient le lien entre les autorités et eux. Le groupe de Tunis était commandé par El-Castalli, El-Haj-Ali Catalina et Mohammad Garmattou. Ces trois-là étaient chargés de rechercher dans la région proche de Tunis un endroit, qui par la géographie, par la pluviométrie, par toutes sortes de conditios était capable de les réunir. Le choix avait été fixé sur Zaghouan.

Aperçu géneral sur les mons de famille:

\section{a) Les ethniques}

Les Sbanioul.

Les Andoulsi.

Les Catlan.

Les Bortguis.

Les Gharnati.

Les Lacanti.

Les Balancianou.

Les Ichbilli.

Les Morsi.

Les Mayourqui.

Les Chalouki (de Sanlùcar de Barrameda au nord-ouest de Cadiz, ou bien de Sanlùcar la Mayor aux environs de Séville).

Les Alcartagenni (de Caragène). 
Les Beji (de Beja, au sud du Portugal).

Les Calasadi (de Calzada de Calatrava, à l'ouest de Baena).

Les Zghonda (de Sagunto, au nord de Valence).

Les Chouria (de Soria).

Les Jhin (de Jaen).

Les Guadachi (de Guadix).

Les Chrichi (de Jerez de la Frontera).

Les Ouichca (de Huesca).

Les Alcastalli.

Les Alhajri (de Guadalajara).

Les Billich (de Velez).

Les Carabaca (de Caravaca).

Les Balma.

Las M'nara (de Almenara au nord de Valence).

Les Berrayana (de Burriana au nord de Sagunto).

Les Cotrane (de Catral dans la province d'Alicante), célèbre famille de relieurs d'art, le dernier descendant est mort dans les années soixante; ils sont de Tunis. Un autre descendant travaillait pendant les années 20/30 au archives officielles de l'Etat.

Les Alkharraz (de Alcaraz au sud-ouest d'Albacete).

Les Bidda (de Ubeda au nord-ouest de Jaen).

Les Hendili (de Alhendin dans la province de Grenade).

Les Taj (de Río Tajo).

Les Bargaoui (de Berga).

Les Nijar (de Nijar, au nord-ouest d'Almeria).

Les Tamarit (de Tamarit de Litera, au sud-est de Huesca, ou bien de Tamarit au nord-est de Tarragona).

Les Skhiri Carmadoune.

Les Terouel (de Teruel).

Les M'kada (de Maqueda).

Les M'nakkbi (de Almuñecar).

Les Mencia (de Mansilla de las Mulas, à l'est de Léon, ou bien Mansilla, au nordouest de Soria).

Les Borji (de Borja au nord-ouest de Zaragoza ou bien de Borjas Blancas au nord de Tarragona).

Les Birra (de Vera).

Les Herrera (de Herrera del Duque, au sud-ouest de Tolede).

Les Selmi (de Medinaceli, à l'extrême sud de Zaragoza, ou bien de Portaceli, à l'ouest de Sagunto).

Les Chalbi (de Sijves, au sud du Portugal, ou bien de Chelva, au nord-ouest de Valence).

Les Gueddana (de Guadiana).

Les Zamouri (de Zamora).

Les Camech (de Camas).

Les Basti (de Baza, au nord d'Almeria).

Les Gueddich (de Cadiz).

Les Ochkor (de Huescar à l'ouest de Zaragoza).

Les Khadhraoui (de Algeciras). 
Les Batarni (de Paterna de Riviera, au sud-est de Cadiz, ou bien de Paterna, aux environs de Valence).

Les lchtibba (de Estepa).

Les Koudia (de Alcoudia de Carlet, au nord-ouest d'Alcira au sud de Valence).

Les Bouguerra (de Bugarra à l'ouest de Sagunto).

Les Belaguer (de Balagues, au nord-est de Lerida).

Les El-khamisi (de Algemesi, au nord d'Alcira).

Les Naifar (de Binefar, au sud-est de Huesca).

b) Les noms chretiens et espagnols

Les Boutricou (de Patric).

Les Bantor (de Pintor).

Les Blayech (de Blaise).

Les Andrech (de Andrés).

Les Ambroch (de Ambroise).

Les Catalina (de Catalina).

Les Madhor (de Amador).

Les Sanchou (de Sancho).

Les Douardalitou (de petit Edouard).

Les Filibou (de Philippo).

Les Ajjourji (de Georges).

Les Christou (de Christ).

Les Gasmir (de Casimir).

Les Gmach (de Gómez).

Les Albaz (de La Paz).

Les Martinech (de Martínez).

Les Rouissi (de Ruiz).

Les Bitri (de Pietri).

Les Bachcouel (de Pascual).

Les Marcou (de Marco).

Les Debbach (de Paz, ou bien de «Dibaj», la soie).

Les Batis (de Batis).

Les Cheniour, les Ben Seniour (de Señor).

Les Louich (de Luis).

Les Lebich (de López).

Les Miquil (de Miguel).

Les Jouane (de Juan).

Les Jbis, Zbiss (de Llopis).

Les Menious (de Muñoz).

Les Ballouma (de Paloma).

Les Ibn-Al-mujira (de la Mujer).

Les Cabisa (de Cabeza).

Les Mira (de Mira).

Les Manita (de Manita, il est dit que dans ce contexte le nom exprime plutôt le manchot, une prothèse de bras...).

Les Sangre (de Sangre).

Les Sabata (de Zapata). 


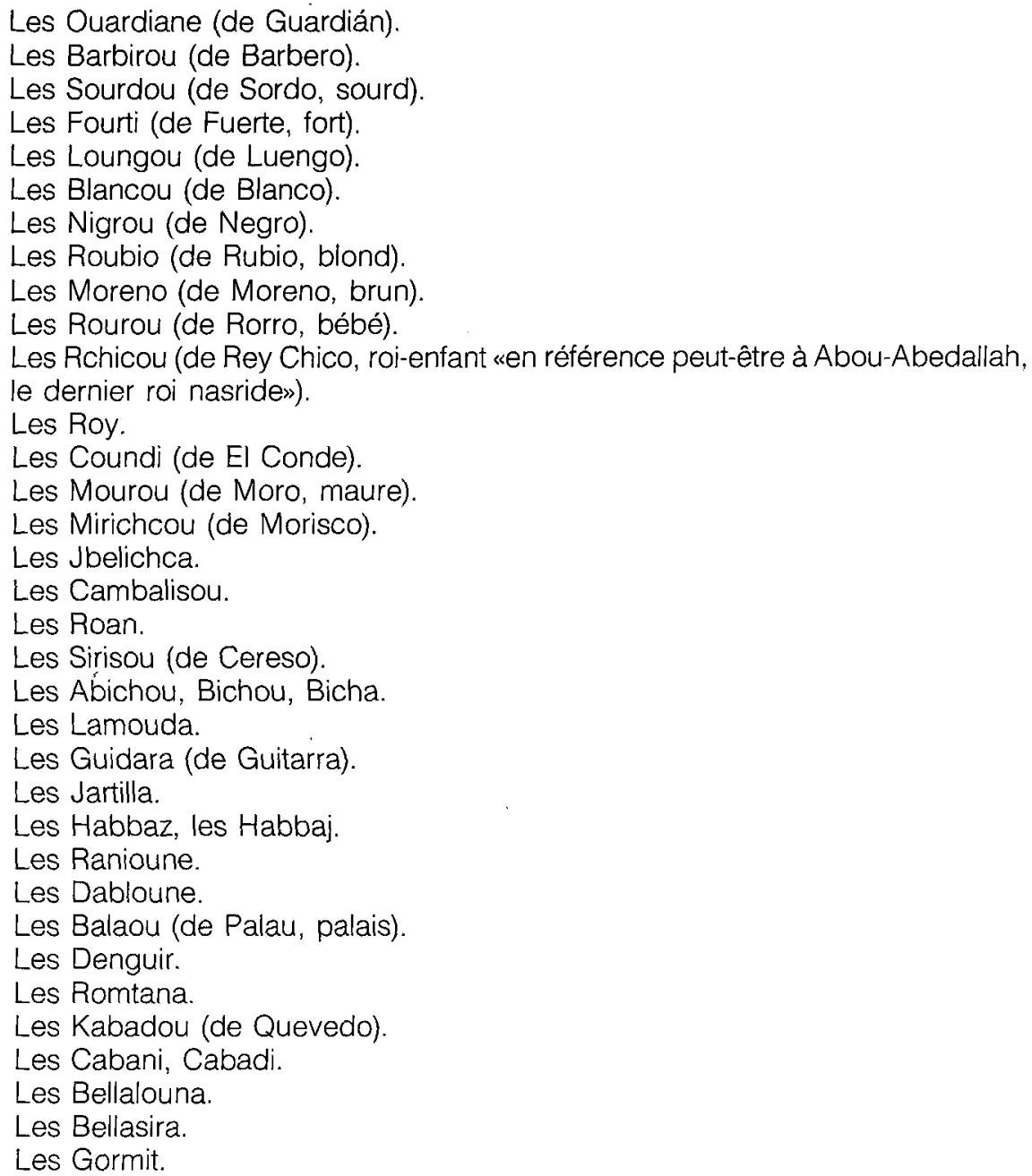

\section{Conclusion}

Voila ce à quoi nous avons abouti, ce sont des propositions que nous présentons. Nous avons pris de sérieuses précautions quant à leur justesse, mais nous avons par devers nous une quantité importante de noms dont nous ne sommes pas sûrs de leur exactitude. Notre effort tendra, nous l'espérons, à les éclairer un peu plus en vue d'un prochain article.

Néanmoins, cet exposé aura servi à montrer combien après plusieurs siècles d'abandon de leur Espagne originelle, les Andalous moriscos ont réussi à conserver intactes des traces de leurs origines et une identité andalouse trés revendiquée jusqu'à nos jours. 